\title{
Antimicrobial and antioxidant linen via laccase-assisted grafting
}

\author{
Carla Silva ${ }^{a}$, Teresa Matamá a,b ${ }^{\text {, SuYeon Kim }}{ }^{\text {a }}$, Jorge Padrão ${ }^{b}$, Endry Nugroho Prasetyo ${ }^{c}$, Tukayi Kudanga $^{c}$, \\ Gibson S. Nyanhongo ${ }^{c}$, Georg M. Guebitz ${ }^{\mathrm{c}}$, Margarida Casal ${ }^{\mathrm{b}}$, Artur Cavaco-Paulo ${ }^{\mathrm{a}, *}$ \\ ${ }^{a}$ University of Minho, Textile Engineering Department, 4800-058 Guimarães, Portugal \\ ${ }^{\mathrm{b}}$ University of Minho, Biology Department, 4710-057 Braga, Portugal \\ ${ }^{\mathrm{c}}$ Graz University of Technology, Institute of Environmental Biotechnology, Petersgasse 12/1, A-8010 Graz, Austria
}

\section{A R T I C L E I N F O}

\section{Article history:}

Received 16 November 2010

Received in revised form 25 March 2011

Accepted 26 March 2011

Available online 2 April 2011

\section{Keywords:}

Chitosan

Catechin

Laccase

Polymerization

Linen

\begin{abstract}
A B S T R A C T
A laccase from Ascomycete myceliophthora thermophila was used to assist the binding of chitosan and catechin onto a previous enzymatically oxidized linen surface. The process consists of the pre-treatment of the linen with laccase followed by the application of chitosan in a first step and catechin plus laccase in a second step. The results presented here support the conclusion that laccase is able to oxidize phenols naturally existing in flax fibres, and that the 0 -quinones formed promote the attachment of chitosan or/and catechin. The pre-treatment of linen with laccase is therefore the key factor for the success of catechin and chitosan grafting. A multifunctional linen product with both antioxidant and antibacterial properties was obtained with an acceptable level of durability in terms of end user requirements.
\end{abstract}

(c) 2011 Elsevier Ltd. All rights reserved.

\section{Introduction}

Flax (Linum usitatissimum L.) fibre, obtained from the stem of plants belonging to the family Linacea is particularly attacked by fungi and bacteria during storage [1]. Microbial growth on a flax textile fabric leads to irreversible changes of a destructive character due to oxidation, hydrolysis and fission of polymer chains. The chemical changes ultimately lead to the material's degradation, loss of strength and elongation, decolorization and changes in appearance [1]. These detrimental effects can be avoided or controlled by a durable antimicrobial and antioxidant finishing of textiles using specific agents [2]. An ideal antimicrobial finishing must satisfy several requirements, of which the most important are a broad spectrum of activity and low toxicity to the consumer [2].

Chitosan is drawing attention as a broad antimicrobial agent innocuous to man. It is the deacetylated derivative of chitin, the main component of the shells of crustaceans [2,3]. The antimicrobial mechanism of chitosan is still not completely understood, but it is generally accepted that the primary amine groups provide positive charges that interact with negatively charged residues on the surface of microbes. This antimicrobial ability, coupled with its non-toxicity, biodegradability and biocompatibility, makes chitosan an eco-friendly product for use in diverse areas, such as food science, agriculture, medicine, pharmaceuticals and textiles [2].

\footnotetext{
* Corresponding author. Tel.: +351 253510271; fax: +351253510293.

E-mail address: artur@det.uminho.pt (A. Cavaco-Paulo).
}

Moreover, it has been shown that chitosan can replace other toxic and not easily degradable chemical products used for the same purpose [3]. Other textile materials, including cotton, silk, nylon, PET, and nonwoven polypropylene (PP) fabrics, have been modified with this compound and excellent antibacterial activities have been reported [4-10].

Due to the natural presence of phenols that can be oxidized and initiate irreversible destructive changes, physical agents can also damage flax fibres and fabrics. Combined with an antimicrobial finishing, flax materials can benefit from an antioxidant functionality, by means of antioxidant deposition. Catechins are the basic structural units of condensed tannins that belong to the class of flavan-3-ols, and they are found in a wide variety of vegetables, herbs and teas. These compounds are protective against cancer, inflammatory and cardiovascular diseases, mainly due to their antioxidant activities and abilities to scavenge free-radicals [11-14].

As polyphenols, catechins are susceptible to enzymatic and non-enzymatic oxidations, giving rise to a variety of dimeric, oligomeric and polymeric products [11,15-17]. Being o-dihydroxylated compounds suggests that they are potential substrates of laccase. Laccase (EC 1.10.3.2) is able to catalyze the $o$-hydroxylation of monophenols to $o$-diphenols and the oxidation of $o$-diphenols to o-quinones. The quinones formed are highly reactive and can undergo nucleophilic attack by other phenolic groups, amines, proteins and peptides [18]. Poly-catechin presents a much higher superoxide scavenging activity than the monomer catechin, making enzyme-catalyzed oxidative polymerization of phenolic compounds an important approach for producing new substances 
with higher antioxidant properties [15]. Many reports have shown that high molecular weight polyphenols exhibit enhanced biological antioxidant properties [19]. Due to their ability to degrade lignin, laccases have been used for the bleaching of flax pulp and cellulose fibres $[20,21]$. Aracri et al. grafted simple phenols on flax and sisal pulp fibres using laccases. This resulted in the increase of pulp kappa number, suggesting the incorporation of the phenols into fibres [22]. Moreover, in recent years the laccase-catalyzed polymerization of phenolic compounds, such as syringic acid, tannins and others, has been exploited [15-17]. In addition to their antioxidant property, many of these phenolic compounds also show antimicrobial activity. It has been reported that lignocellulosic substrates, as well as wool, when treated with simple phenols or their oligomeric coupling products in the presence of laccase, show improved antibacterial resistance towards Gram-positive and Gram-negative bacteria [23,24].

Antimicrobial and antioxidant agents can be effectively grafted onto lignocellulosic materials to prevent biodeterioration and oxidation, however grafting must fulfil some criteria, such as higher resistance to water exposure.

The main objective of this study is to produce linen that is more resistant to biodeterioration and oxidation by laccase-assisted grafting of chitosan and catechin. Phenolic subunits naturally present in lignin can react with laccase via a one-electron oxidation, followed by further enzymatic or non-enzymatic reactions of the formed radical [25]. This makes linen an excellent candidate for laccase-assisted grafting of phenolics. The innovative contribution of this study is the grafting of products onto a previous laccase oxidized surface. Laccase acts as a key factor in the first stage of the process, oxidizing the phenolic compounds naturally present in linen, which will contribute to a better attachment of chitosan and catechin in a second stage. A multifunctional product with both antimicrobial and antioxidant properties is achieved, with an acceptable level of durability in terms of end user requirements.

\section{Experimental}

\subsection{Materials and reagents}

Laccase from the Ascomycete myceliophthora thermophila, Novozym ${ }^{\circledR} 51003$ (306 $\mathrm{U} \mathrm{mL}^{-1}$ at $25^{\circ} \mathrm{C}, 1667 \mathrm{U} \mathrm{mL}^{-1}$ at $50{ }^{\circ} \mathrm{C}$ ), was obtained from Novozymes (Bagsvaerd, Denmark). The 100\% raw linen, with a $14 / 14$ (warp/weft) $\mathrm{cm}^{-1}$ yarn count, was kindly supplied by the "Institute of Natural Fibres" (Poland). Practical grade chitosan from shrimp shells, with a $\geqslant 75 \%$ deacetylation degree, and catechin hydrate were obtained from Sigma (St. Louis, USA). All the other chemicals used in this study were purchased from Sigma (St. Louis, USA), and used without further purification.

\subsection{Enzymatic oxidation of linen}

In order to evaluate the influence of previous fabric oxidation by laccase, the experiments were performed on linen samples with and without previous enzyme treatment. The enzymatic pre-treatment consisted of an overnight incubation of fabric samples with $1 \mathrm{U}$ of laccase/g of fabric in acetate buffer $(50 \mathrm{mM}) \mathrm{pH} 5$ at $50^{\circ} \mathrm{C}$. The fabrics were washed with a Lutensol AT25 solution $\left(2 \mathrm{~g} \mathrm{~L}^{-1}\right)$, and then with distilled water to remove all the protein bound to the fabric surface. Linen samples did not present different coloration after laccase oxidation, maintaining original color aspect.

\subsection{Optimization of catechin grafting onto linen surface}

The first parameter to be optimized was the laccase concentration for the catechin grafting. For each enzyme concentration $(2,3$,
$5,7,8,17,84$ and $\left.167 \mathrm{U} \mathrm{mL}^{-1}\right)$, the catechin amount used was $5 \mathrm{mM}$ in acetate buffer $(50 \mathrm{mM})$ at $\mathrm{pH}=5$. In each pot of a certain laccase concentration, two linen samples were introduced: one was preoxidized with laccase (according to Section 2.2) and the other was without any previous treatment. The incubation period was $24 \mathrm{~h}$ at $50^{\circ} \mathrm{C}$. The samples were then washed with a detergent solution ( $2 \mathrm{~g} \mathrm{~L}^{-1}$ Lutensol AT25) to remove all the catechin noncovalently attached to the fabric.

The second parameter to be optimized was the catechin concentration, using the most convenient laccase activity determined, as described above. The tested concentrations were 1, 2, 5, 10 and $20 \mathrm{mM}$. The incubation procedure was the same as described above. All the measurements were performed using duplicate samples.

\subsection{Linen coating with chitosan}

Several concentrations of chitosan $(5,10$ and $50 \mathrm{mM})$, previously dissolved in $1 \%$ acetic acid, were applied onto linen. In each pot of a certain chitosan concentration, two linen samples were introduced: one was preoxidized with laccase (according to Section 2.2) and the other was without any previous treatment. The incubation period was $4 \mathrm{~h}$ at $60^{\circ} \mathrm{C}$. The samples were rinsed with an acetic acid solution $(10 \mathrm{mM})$ to remove unlinked chitosan. The samples were then washed with a detergent solution $\left(2 \mathrm{~g} \mathrm{~L}^{-1}\right.$ of Lutensol AT25). All the measurements were performed using duplicate samples.

\subsection{Chitosan and catechin grafting onto linen surface}

Chitosan and catechin were applied sequentially to linen samples previously oxidized with laccase (see Section 2.2) and not pre-treated. In a first step, linen samples were incubated overnight with $10 \mathrm{mM}$ chitosan in acetate buffer $(\mathrm{pH} 6)$ at $50^{\circ} \mathrm{C}$. Afterwards, the samples were washed with a $10 \mathrm{mM}$ acetic acid solution, in order to remove all the non-fixed chitosan. In a second step, the cationized linen samples were incubated overnight with $5 \mathrm{mM}$ catechin in acetate buffer $(50 \mathrm{mM}), \mathrm{pH} 5$, in the presence of $2 \mathrm{U} \mathrm{mL}^{-1}$ laccase, at $50{ }^{\circ} \mathrm{C}$.

\subsection{Characterisation of the laccase oxidation products in solution}

To monitor the polymerization of catechin by laccase oxidation, the spectra of each prepared solution was measured in a UV/visible spectrophotometer using a diode-array J\&M Tidas spectrophotometer (J\&M Analytische Mess und Regeltechnik GmbH, Germany), before and after enzymatic oxidation.

\subsection{Characterisation of linen grafted with chitosan and catechin}

\subsubsection{Scanning electron microscopy (SEM)}

The grafted fabrics were scanned in different places of the sample using an electron microscope model LEICA S360 with a backscattered and secondary electrons detector, at 2000x magnification. The samples were coated with a thick layer of gold before scanning with SEM and all the images were carefully taken using the same sample position of weft and warp.

\subsubsection{Catechin detection by visible spectroscopy}

After catechin polymerization and grafting, linen samples present color with a maximum absorption peak at $360 \mathrm{~nm}$. The color measurement was performed using the spectrophotometer Spectraflash 600 Plus at illuminant $\mathrm{D}_{65}$ (Datacolor International). The color strength was evaluated as $\mathrm{k} / \mathrm{s}$ at the maximum absorption wavelength $360 \mathrm{~nm}$. The ratio between absorption coefficient $(k)$ and scattering coefficient $(s)$ is related to reflectance data by 
applying Kubelka-Munk's law at each wavelength, and is proportional to catechin concentration. The color difference between grafted and non-grafted samples was obtained by the relation;

$\frac{k / s_{\text {grafted }}-k / s_{\text {non-grafted }}}{k / s_{\text {non-grafted }}} \times 100$

All measurements were performed using at least triplicate samples.

\subsubsection{Chitosan detection - qualitative determination by reactive dyeing}

Linen samples previously oxidized with laccase and coated with chitosan were subjected to a specific staining with a reactive dye in order to measure the coating efficiency. The dye used was Lanasol Red 66, a wool reactive dye, which reacts specifically with amino groups. The more chitosan that attaches to the fabric surface, the more amino groups are available to react with Lanasol Red 66, and consequently the more colored the fabric is. The color measurements were carried out as described in Section 2.7.2, at the maximum wavelength $570 \mathrm{~nm}$. All measurements were performed using at least triplicate samples.

\subsubsection{Washing fastness}

Washing fastness evaluation of grafted linen samples was performed using the NP EN ISO 105-C06:1999 standard method. The method is based on the washing of the samples coupled to a testimony sample that is evaluated in terms of staining using the grey scale as the measuring tool. The level of staining reveals the washing resistance of our main sample, ranging from 1 for poor resistance and up to 5 for excellent resistance. All the measurements were performed using duplicate samples.

\subsubsection{Rubbing fastness}

Rubbing fastness evaluation of grafted linen samples was performed using the ISO 105-X02:1993 standard method. This methodology consists of the rubbing of the samples against a cotton testimony. The level of rubbing fastness is also evaluated using the grey scale as reference. All the measurements were performed using duplicate samples.

\subsubsection{Light fastness}

The light fastness of grafted linen samples was determined by using the Accelerated Weathering Tester (QUV) Spray LU-0819 from Q-Panel (UVA 340 lamp at $70^{\circ} \mathrm{C}$ and $77 \mathrm{~W} / \mathrm{m}^{2}, 32 \mathrm{~h}$ of exposure). The light fastness was, in this case, evaluated in terms of color remaining after light exposure. The color strength of the samples was measured before and after UV exposition. All the measurements were performed using duplicate samples.

\subsubsection{Tensile properties of fabrics: determination of maximum force and elongation}

Maximum force and elongation of grafted linen samples were measured with a Housfield dinamometer according to the NP EN ISO 13934-1:2001 - Tensile properties of fabrics Part 1: determination of maximum force and elongation at maximum force using the strip method.

\subsubsection{Antioxidant activity}

The antioxidant activity of grafted linen samples was measured in terms of tetramethoxy azobismethylene quinone (TMAMQ) reduction ( $1 \mu \mathrm{M}$ TMAMQ reduced by $1 \mu \mathrm{M}$ antioxidant) as previously described by Nugroho Prasetyo et al. [26] TMAMQ was produced as previously described [26,27]. Briefly, syringaldazine $(0.17 \mathrm{mM})$ was incubated with $50 \mu \mathrm{L}$ of laccase $\left(1.2 \mathrm{U} \mathrm{mL}^{-1}\right.$ determined as previously described by Nugroho Prasetyo et al. [26]) in $50 \mathrm{mM}$ sodium citrate buffer at $\mathrm{pH} 4.5$ for $10 \mathrm{~min}$ at $30^{\circ} \mathrm{C}$ while shaking at $140 \mathrm{rpm}$ using a Thermomixer (Eppendorf AG, Germany). The reaction was then immediately stopped by freezing in liquid nitrogen followed by lyophilization using Labconco Freeze Dry System/FreeZone $\odot 4.5$ L Benchtop Model 77500 (Vienna, Austria). The freeze drier was operated at a temperature of $-48^{\circ} \mathrm{C}$ and at a vacuum pressure of $3 \times 10^{-4}$ mbar. The freeze-dried TMAMQ was then dissolved in ice-cold methanol and centrifuged at $13,000 \mathrm{rpm}$ to precipitate the enzyme. The resulting supernatant containing TMAMQ was used for the antioxidant activity assay. The antioxidant activity of the polyphenol grafted fabrics $(1 \times 2 \mathrm{~cm})$ was measured by immersing the fabric pieces into a solution containing TMAMQ and incubating at $30^{\circ} \mathrm{C}$ for $30 \mathrm{~min}$. After removing the fabric the change in absorbance was monitored at $530 \mathrm{~nm}$, as previously described by Nugroho Prasetyo et al. [26]. In this study, the methodology described was applied on flax fabric substrates for the first time.

\subsubsection{Antimicrobial activity}

The antibacterial activity of grafted linen samples was evaluated according to the "AATCC Test Method 100-1999, Antibacterial Finishes on Textile Materials: Assessment of". The Gram positive Staphylococcus aureus, American Type Culture Collection 6538, and the Gram negative Escherichia coli HB101 were chosen as test bacteria. The fabric swatches were previously sterilized under UV; two swatches were used for each assay condition and inoculated with $1 \mathrm{~mL}$ of a cell suspension prepared by diluting the pre-inoculum in PBS to the following concentrations: $6.7 \times 10^{7} \mathrm{col}-$ ony forming units (CFUs)/mL for S. aureus and $6.9 \times 10^{8}$ CFUs $/ \mathrm{mL}$ for E. coli.

\section{Results and discussion}

\subsection{Polycatechin evaluation by UV-vis spectrophotometry}

According to the literature and based on the results of UV-visible spectrophotometry, the products of laccase-catalyzed oxidation of catechin were identified $[28,29]$. Two mechanisms have been proposed for the formation of catechin dimers: (a) nucleophilic addition of the A-ring of a catechin to the B-ring of its oxidation product (the quinone) and (b) coupling of radicals produced from catechin oxidation. Values that have already been published suggest that laccase-catalyzed polymerization reaction proceeds mainly through the nucleophilic rather than radical mechanism [16].

Fig. 1 shows the UV-vis spectra of the colored products obtained from the enzymatic oxidation end products of catechin by laccase. The significant absorption band near $280 \mathrm{~nm}$ has been frequently cited as representative of flavonoids (black line). After laccase oxidation, new peaks appeared at $310 \mathrm{~nm}$ and $370 \mathrm{~nm}$ corresponding to catechin polymerization (light grey line). The increased absorption band within the visible area, after laccase oxidation, was observed and might be linked to the polymerization event or crosslinking reaction. During incubation, the peak at $370 \mathrm{~nm}$ was shifted and the peak intensity became larger (dark grey line). These events are related to the laccase-catalyzed formation of poly-catechin. The increase in the absorbance at $650 \mathrm{~nm}$ corresponds to a color increase during the oxidation process from uncolored to dark orange.

\subsection{Optimization and evaluation of catechin and chitosan grafting}

3.2.1. Effect of previous linen enzymatic oxidation in catechin grafting

Laccase is known for its ability to degrade lignin and catalyze the oxidation of phenolic groups. Phenolic subunits in lignin can react with laccase via one-electron oxidation, followed by further enzymatic or non-enzymatic reactions of the newly formed radicals. 


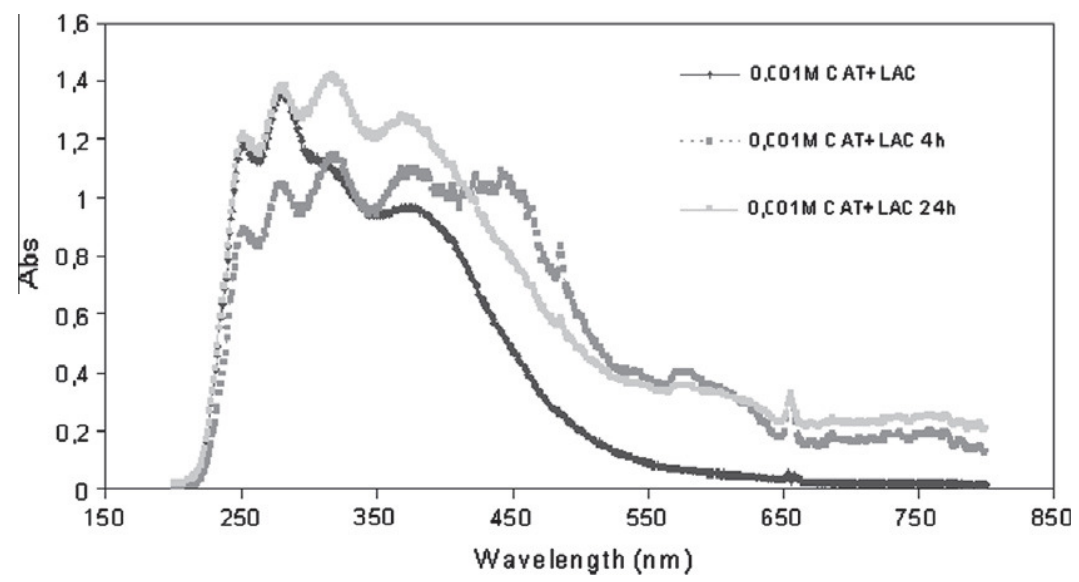

Fig. 1. Modification of the UV-visible spectrum of catechin in solution during the laccase-catalyzed polymerization $\left(\mathrm{pH}=5 ; 50{ }^{\circ} \mathrm{C} ; 24 \mathrm{~h} ; 2 \mathrm{U} \mathrm{mL}^{-1}\right.$ laccase).

Before optimization of the Ascomycete myceliophthora thermophila laccase-catalyzed oxidation of catechin and consequent polymerization onto the linen surface, some experiments were performed to verify if it was beneficial for the catechin grafting to have linen previously oxidized by the same enzyme. In this previous treatment, it was expected that laccase would be able to cleave some of the bonds in lignin and catalyze the oxidation of naturally occurring phenolic subunits, which would facilitate the subsequent fixation of catechin. The results clearly demonstrate that flax substrates previously oxidized by laccase present higher color strength, which is directly related to the concentration of catechin grafted onto the surface (Fig. 2). The pre-treatment is in fact essential to achieve high levels of catechin grafting in linen substrates.

\subsubsection{Optimization of catechin grafting conditions}

Several concentrations of laccase were used to perform the first optimization experiments, where a concentration of catechin was fixed ( $5 \mathrm{mM}$ ) (Fig. $3 \mathrm{~A}) .2 \mathrm{U} \mathrm{mL}^{-1}$ of laccase was found to be the most promising concentration for use in further experimentations. Therefore, this value was fixed and other assays were performed with varying concentrations of the phenolic compound. Higher levels of grafting are obtained for the use of higher concentrations of the phenolic compound; however, a higher level of staining is associated with this finding. Taking into account this outcome, a concentration of catechin between $2 \mathrm{mM}$ and $5 \mathrm{mM}$ can be used to achieve the higher levels of grafting without staining (Fig. 3B). The above results confirm that laccase can oxidize the surface of flax fibres and convert some existing phenolic groups into reactive

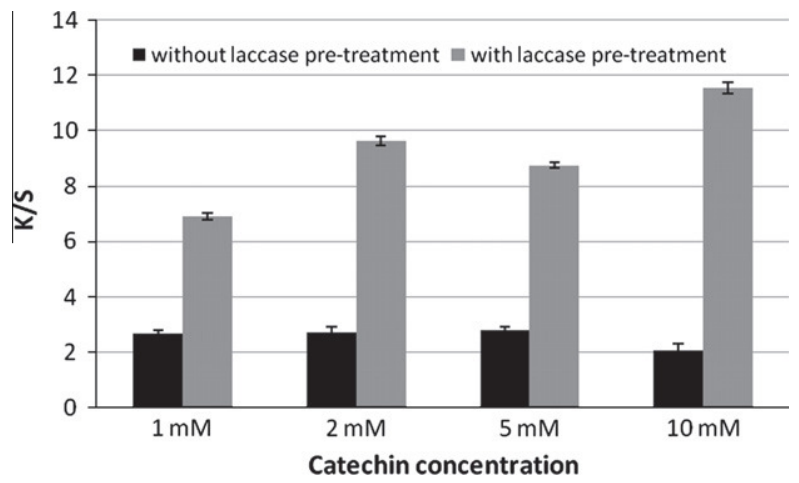

Fig. 2. Color strength $(k / s, \lambda=360 \mathrm{~nm})$ of linen samples, with or without previous treatment, coated with different catechin concentrations in the presence of $2 \mathrm{U} \mathrm{mL}^{-1}$ laccase. o-quinones. These species may undergo a complex set of non-enzymatic reactions with nucleophiles, such as free amino or $\mathrm{OH}$ groups. The chemistry of the reaction is not well understood, but it is expected that quinones are covalently conjugated to free amine or other phenolic groups through the Schiff-base and/or Michael-type reaction mechanisms [9]. The results also provide evidence that samples previously oxidized by laccase present higher catechin grafting probably due to the higher amount of quinones available to react with other phenols like catechin.

\subsubsection{Effect of previous linen oxidation in chitosan coating}

After catechin grafting optimization, the chitosan application was tested. To evaluate the efficiency of chitosan coating, a methodology to detect this compound at the surface of the linen samples was needed. Therefore, a staining methodology with a
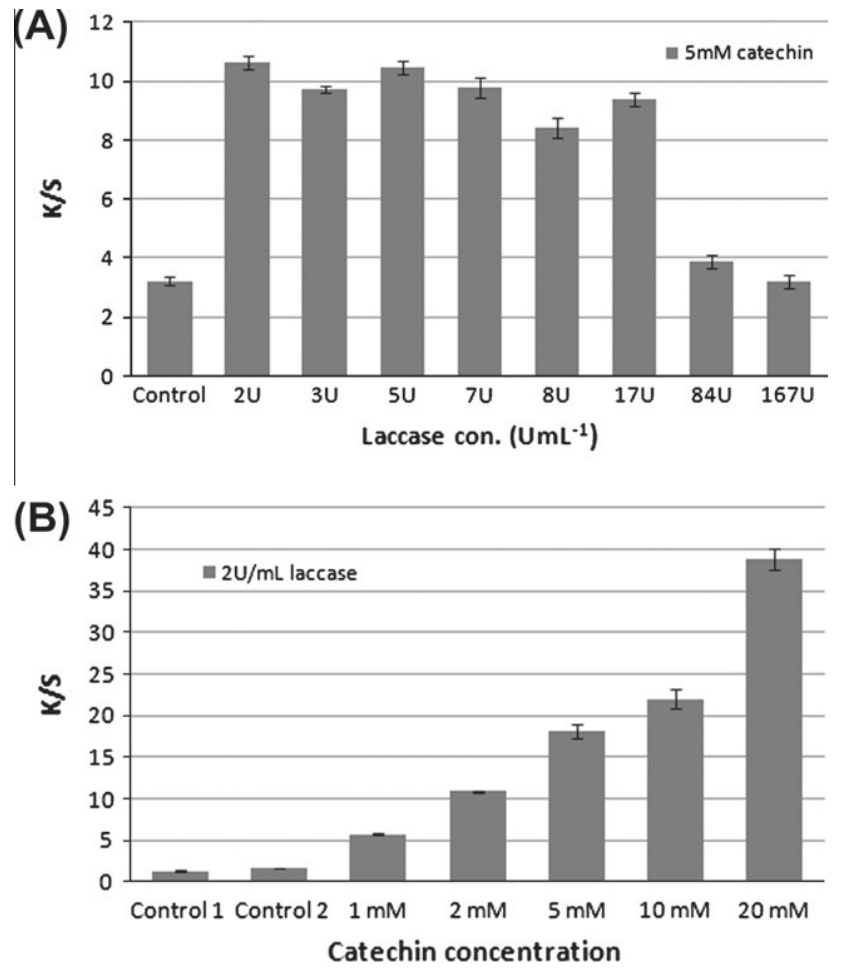

Fig. 3. Color strength $(k / s, \lambda=360 \mathrm{~nm})$ of linen samples incubated with different concentrations of laccase $\left(5 \mathrm{mM}\right.$ catechin; $\left.50^{\circ} \mathrm{C}, \mathrm{pH}=5,24 \mathrm{~h}\right)(\mathrm{A})$; color strength of linen samples incubated with different concentrations of catechin $\left(2 \mathrm{U} \mathrm{mL}^{-1}\right.$ laccase; $50^{\circ} \mathrm{C} ; \mathrm{pH}=5,24 \mathrm{~h}$; control 1: enzyme/no catechin; control 2: catechin/ no enzyme) (B). 


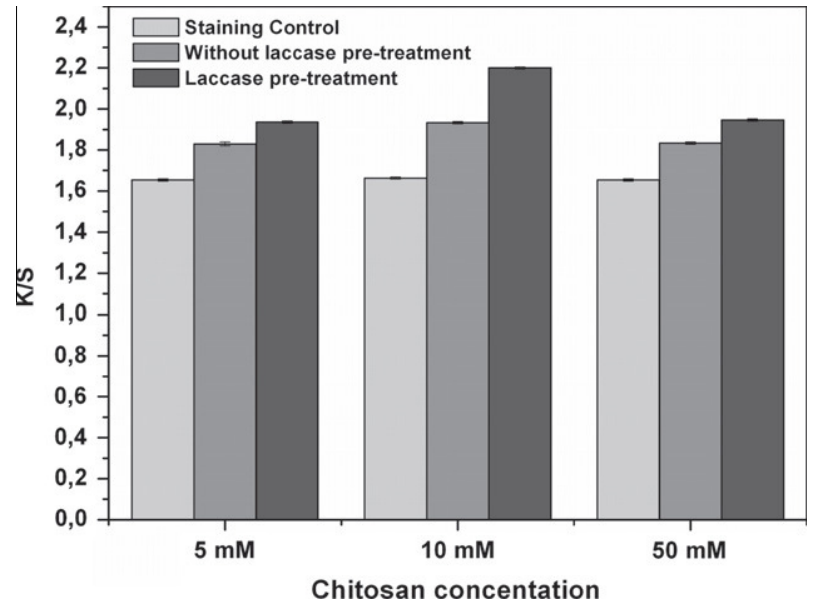

Fig. 4. Color strength $(k / s, \lambda=570 \mathrm{~nm})$ of linen samples coated with chitosan and stained with a reactive dye (Lanasol Red 66 ) for chitosan detection.

wool reactive dye (Lanasol Red 66), which is able to react covalently with amino groups, was performed. The values of color strength are proportional to the concentration of dye at the surface of the fabric. The concentration of dye increases due to the presence of amino groups from chitosan on the linen surface.

Fig. 4 shows the results of color strength (in terms of $k / s$ values) for three independent stainings. In each pot of dye, a control consisting of a piece of linen that had not undergone chitosan treatment was included. As the results show, these controls gave equal $\mathrm{k} / \mathrm{s}$ values in all three of the independent stainings. The $\mathrm{k} / \mathrm{s}$ value around 1.6 corresponds to the natural linen color. Lanasol was not able to react with the linen without chitosan. In each pot of dye, a competitive staining was performed between linen samples grafted with the same amount of chitosan but differing only in the pre-treatment plus the control. The effect of laccase pre-oxidation of flax substrates is significant, according to the $\mathrm{k} / \mathrm{s}$ levels obtained. At each chitosan concentration, the level of chitosan attachment was higher for previously oxidized samples. The oxidation led to an increase of possible chitosan binding sites, which were saturated because the highest chitosan concentration did not lead to a higher $k / s$ value. The best results were obtained when $10 \mathrm{mM}$ of chitosan was applied.

\subsection{Characterisation of grafted linen fabrics}

\subsubsection{Scanning electron microscopy}

The SEM microphotographs clearly show the deposition of the polymers used in this study. The flax fibres morphology became more irregular with catechin and chitosan (Fig. 5B), just chitosan (Fig. 5C) or just catechin (Fig. 5D) treatments compared with the untreated control (Fig. 5A).

\subsubsection{Fastness properties (washing and rubbing)}

Durability is the main concern of today's Textile Industry regarding antibacterial finishing. Textiles are subjected to frequent washing, rubbing and perspiration during their usage. The durability of the finish applied on the textile material to these conditions is therefore extremely important. Table 1 summarizes the fastness properties of linen samples treated with chitosan and catechin. The
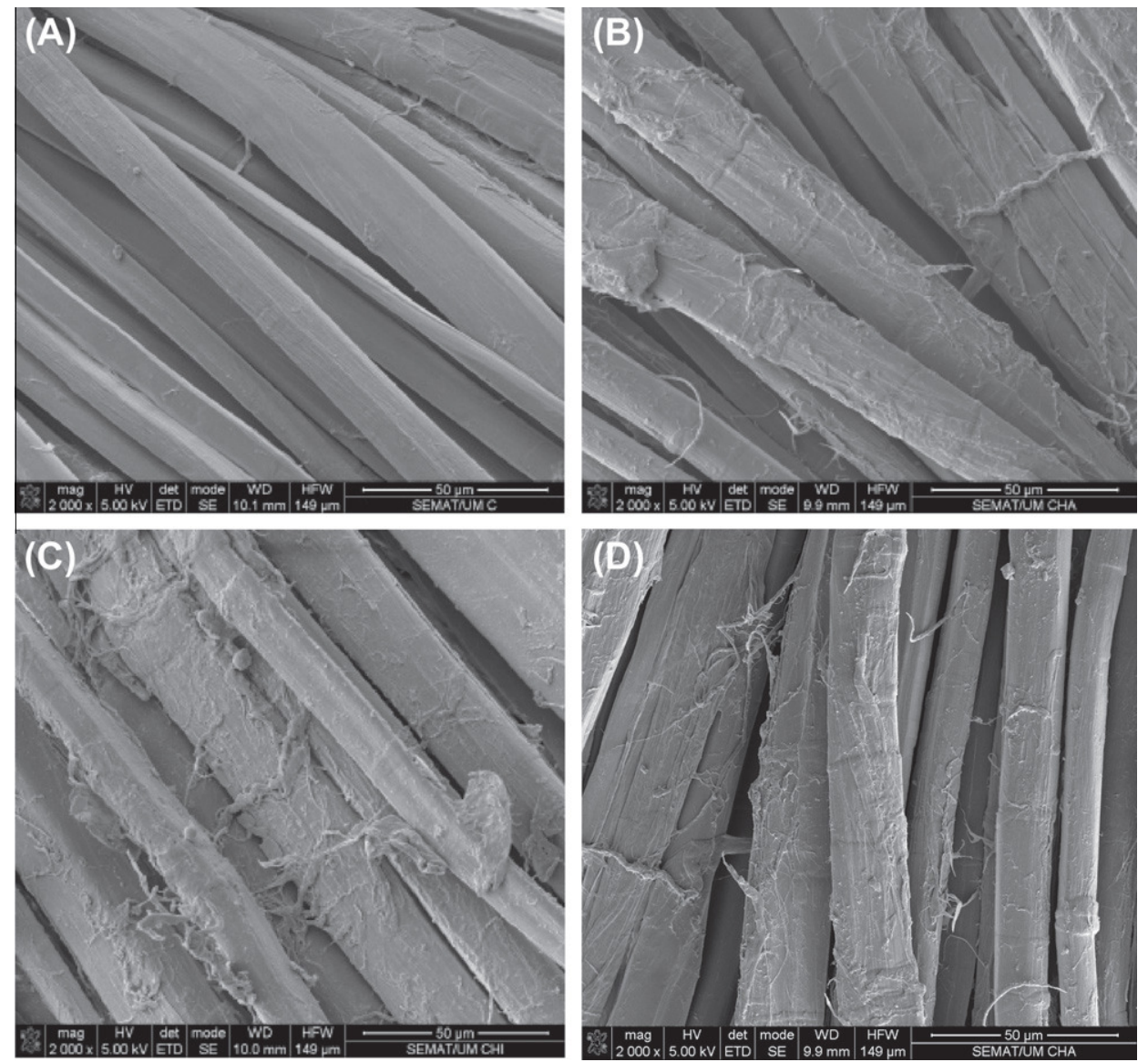

Fig. 5. Scanning electron microscopy images of linen fabrics: (A) control, (B) sample coated with chitosan and catechin amplified, (C) sample coated with chitosan amplified and (D) sample coated with catechin; all the images were amplified $2000 \times$. 
Table 1

Fastness properties of linen samples treated with different concentrations of catechin and chitosan.

\begin{tabular}{|c|c|c|c|c|c|c|c|c|}
\hline & \multicolumn{6}{|c|}{ Washing fastness } & \multicolumn{2}{|c|}{ Rubbing fastness } \\
\hline & Wool & Acrylic & PES & PA & Cotton & Acetate & Dry & Wet \\
\hline Catechin (1 mM) & - & - & - & - & - & - & 5 & $3-4$ \\
\hline Catechin (2 mM) & - & - & - & - & - & - & 5 & 3 \\
\hline Catechin (5 mM) & $3-4$ & $4-5$ & 4 & $3-4$ & 3 & 3 & 5 & 3 \\
\hline Catechin $(10 \mathrm{mM})$ & 3 & 3 & 3 & 4 & 3 & 3 & 5 & 3 \\
\hline Chitosan (5 mM) & $4-5$ & $4-5$ & $4-5$ & 4 & 4 & 4 & - & - \\
\hline Chitosan (10 mM) & $4-5$ & $4-5$ & $4-5$ & $4-5$ & 4 & 4 & - & - \\
\hline Chitosan/catechin (5 mM each) & $2-3$ & 2 & $2-3$ & 2 & 3 & 2 & - & - \\
\hline
\end{tabular}

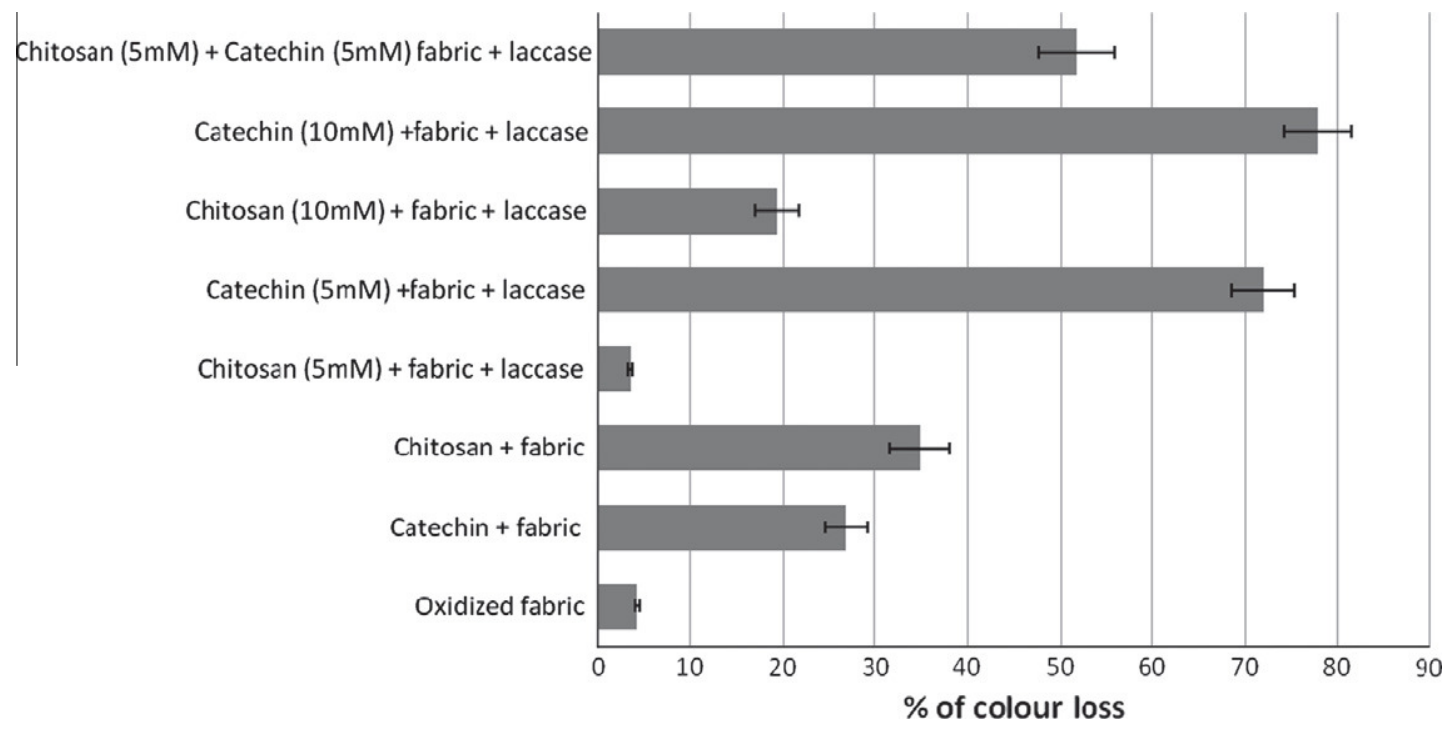

Fig. 6. Light fastness of linen samples coated with chitosan and catechin.

Table 2

Tensile strength and elongation of linen samples after grafting with chitosan/catechin and exposure to UV light.

\begin{tabular}{lll}
\hline & Force $(\mathrm{N})$ & Elongation $(\%)$ \\
\hline Before UV exposure & 870 & 10 \\
After UV exposure & 539 & 16 \\
Variation (\%) & -38 & +60 \\
\hline
\end{tabular}

results demonstrate that, after catechin grafting, the fastness level was good (level 5) for the dry state. For the wet state, the level of fastness decreased but maintained acceptable values in terms of textile acceptance (level 3). When the fabrics were washed after catechin grafting, a huge amount of polymer was leached, though an acceptable level of grafting was achieved as demonstrated by visual analysis (data not shown) and $k / s$ measurement (Fig. 3). Wash-

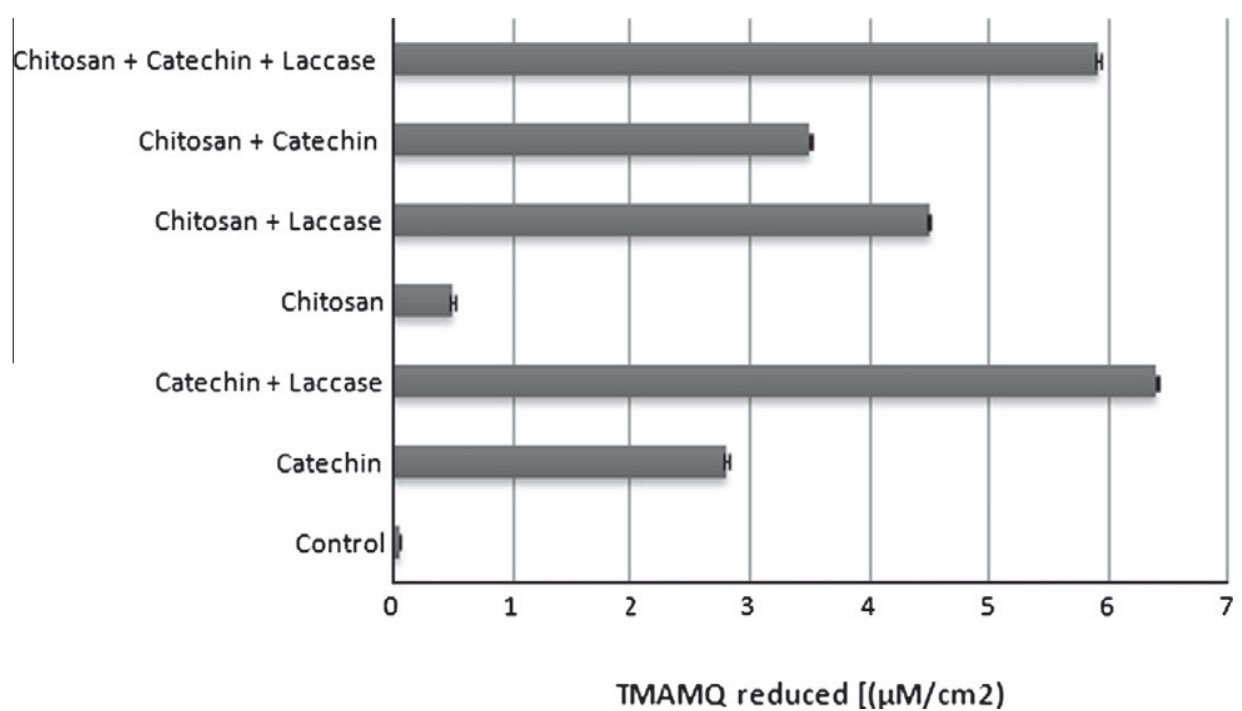

Fig. 7. Antioxidant activity measured in terms of tetramethoxy azobismethylene quinone (TMAMQ) reduction per $\mathrm{cm}^{2}$ of fabric. 


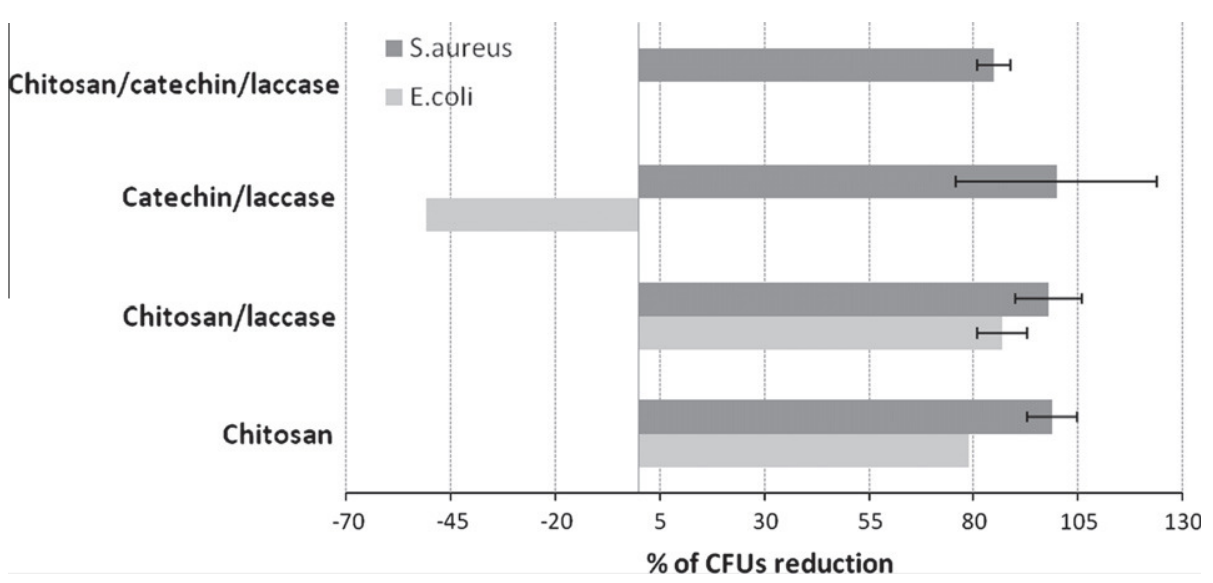

Fig. 8. Antibacterial activity of linen samples coated with chitosan and catechin evaluated according to the AATCC Test Method 100-1999.

ing fastness values were also good (level 4-5) when only chitosan was applied. For the samples grafted with catechin and chitosan in different stages, the fastness levels are acceptable (level 2-3), although lower than those obtained for catechin alone. This might be due to the different supports which catechin attaches to. One is lignin grafted with chitosan and the other is just lignin. When chitosan is present, the fixation of catechin is not so strong.

\subsubsection{Light fastness}

The grafting of catechin gives a yellow coloration to flax substrates. The level of grafting is proportional to the color intensity at the surface of the fabrics. One of the factors to take into account about color durability is resistance to light. The samples were exposed to UVA 340 lamp at $70^{\circ} \mathrm{C}$ and $77 \mathrm{~W} / \mathrm{m}^{2}$ for $32 \mathrm{~h}$ and the light fastness was evaluated in terms of \% of color loss. Fig. 6 reveals that higher color failure is observed on samples grafted with the highest concentrations of catechin $(0.005 \mathrm{M}$ and $0.01 \mathrm{M})$ in presence of laccase. The polymer attached to the linen surface has relatively low resistance to light degradation. When chitosan was applied in a first stage, the poly-catechin formed upon it is more stabilized showing higher light resistance behaviour.

The study of tensile strength and elongation, presented in Table 2 , revealed that after light exposure, the samples present lower resistance to break, showing a variation of $38 \%$. On the other hand, the elongation of the sample increased $60 \%$, which might be attributed to two factors. One is related to the weight gain at the moment of chitosan and catechin grafting and the other can be attributed to the formation of strong bonds between chitosan and/or catechin and the flax fibre surface. Polycatechin might in these cases act as a UV protector. Values of tensile strength of samples grafted with catechin and chitosan alone are not presented since there is no deposition of both products in the absence of laccase.

\subsubsection{Antioxidant activity}

There are several studies about antioxidant activity that consider chitosan and water-soluble chitosan derivatives to be hydroxyl radical scavengers [30] and to have metal-bonding capacities [31,32]. The chitosan antioxidant activity has been explained by several mechanisms [32,33]. One is a free-radical scavenging activity, in which chitosan may eliminate various free-radicals by the action of nitrogen on the $\mathrm{C}-2$ position. The scavenging activities of chitosan derivatives against $\mathrm{OH}$ may be derived from some or all the following: (i) the hydroxyl groups in the polysaccharide unit can react with $\mathrm{OH}$ by the typical $\mathrm{H}$-abstraction reaction; (ii) $\cdot \mathrm{OH}$ can react with the residual free amino groups $\mathrm{NH}_{2}$ to form stable macromolecules radicals; (iii) the $\mathrm{NH}_{2}$ groups can form ammonium groups $\mathrm{NH}_{3}^{+}$by absorbing hydron from the solution, then reacting with ${ }^{\circ} \mathrm{OH}$ through addition reactions.
Linen samples, previously treated with laccase, were grafted under different conditions with catechin and chitosan and analyzed in terms of their antioxidant activities. This property was evaluated by calculating the tetramethoxy azobismethylene quinone (TMAMQ) reduction and the results are presented in Fig. 7. It can be inferred that the presence of laccase during the samples grafting improved the antioxidant activity of linen whatever the compound used (catechin, chitosan or both). The beneficial effect of laccase could be attributed to the formation of chemical bonds that promoted the stable attachment of catechin and chitosan to linen, with more molecules to scavenge the radicals (amount effect). The formation of chemical bonds by laccase can also create more extended and efficient chemical systems for radical scavenging, as would be expected with catechin polymerization. The grafting of flavonoids like catechin and/or poly-catechin onto linen substrates enhances their antioxidant function when compared with the unmodified samples. As a flavonol, catechin has the 2-3double-bond conjugated with a 4-oxo function in its structure. This feature makes catechin a potent antioxidant with its double bonds allowing delocalization of electrons across the molecule, thus stabilizing the phenoxy radical. The results demonstrate that polycatechin possesses a more effective scavenging activity against radicals than that of a monomeric catechin (Fig. 7).

Fig. 7 demonstrates that the grafting of both chitosan and catechin did not have an additive effect on the antioxidant activity when laccase was present. The reduction of TMAMQ is slightly lower when compared with the samples grafted with laccase-catalyzed catechin. The radical-scavenging activity of chitosan was probably affected by the chemical binding of catechin, which could have restricted the exposure of the amine groups and hence led to the lower radical-scavenging activity. Another possibility is that the binding of catechin to chitosan could also have affected the radical-scavenging activity of catechin.

These results disclose not only a new methodology to evaluate antioxidant activity on flax substrates but also evidence that catechin and chitosan were able to increment antioxidant activity of linen. Moreover, laccase was able to catalyze the polymerization of catechin and concomitantly increment its scavenging activity, but when chitosan was present it did not improve the antioxidant properties of linen any further.

\subsubsection{Antimicrobial activity}

The use of flavonoids as potent therapeutics has been well documented [34,35]. Several studies have reported that antibacterial activity of flavonoids is related to damage of the bacterial membranes causing an increase in the permeability of the inner bacterial membrane, and dissipation of the membrane potential. Other 
groups have reported a common structural feature that is related to hydroxylation of the B-ring. The mechanisms of action of the flavan-3-ol (catechin) are related to the damage of the bacterial membranes and to the interactions of several proteins $[35,36]$.

Antibacterial activity of linen samples grafted with both chitosan and catechin was tested against an example of Gram positive (S. aureus) and Gram negative (E. coli) bacterium. As expected, E. coli only showed susceptibility to chitosan treated samples of about $80 \%$ CFUs reduction, either with or without laccase (Fig. 8). Several mechanisms for microbial inhibition by chitosan have been proposed, but the exact mechanism is still not known. The most widely accepted is the interaction of the positively charged chitosan with the negatively charged residues at the cell surface of bacteria, which causes extensive cell surface alterations and alters cell permeability. As a result, chitosan inhibits the normal metabolism of microorganisms and finally leads to the death of these cells. When catechin was present on the samples, alone or conjugated with chitosan, there was no reduction in E. coli CFUs. The low catechin susceptibility of Gram-negative bacteria is attributed to the presence of lipopolysaccharide (LPS) acting as a protective barrier against it [18,37]. Regarding this bacterium, chitosan action was hindered when catechin was present in the sample (Fig. 8). S. aureus was equally sensitive to chitosan and catechin actions.

\section{Conclusions}

The present work demonstrates for the first time that laccase can be used to graft flavonoids and chitosan onto natural fibres like linen. Laccase oxidized surfaces presented higher grafting levels with enhanced washing durability. The results here presented support the conclusion that laccase is able to oxidize flavonoids naturally existing in flax fibres, and that the o-quinones formed covalently bind to the amino groups of chitosan or/and to catechin. The presence of laccase in the medium is therefore the key factor for the success of catechin and chitosan grafting. Laccase acts in a first stage of the process, thus improving the subsequent chitosan and catechin attachment.

The multifunctional linen product achieved presents antioxidant and antimicrobial activities with acceptable levels of grafting durability as confirmed by the fastness tests.

This study opens up a novel strategy for the development of new bioactive textile material.

\section{Acknowledgement}

Carla Silva would like to acknowledge the Portuguese Fundação para a Ciência e a Tecnologia (FCT) for funding under the scholarship SFRH/BPD/46515/2008.

\section{References}

[1] J. Szostak-Kotowa, International Biodeterioration and Biodegradation 53 (2004) 165-170.
[2] Yuan Gao, R. Cranston, Textile Research Journal 78 (2008) 60-72.

[3] S.H. Lim, S.M. Hudson, Polymer Reviews 43 (2003) 223-269.

[4] S.H. Lim, S.M. Hudson, Carbohydrate Polymers 56 (2004) 227-234.

[5] W. Ye, J.H. Xin, P. Li, K.L.D. Lee, T.L. Kwong, Journal of Applied Polymer Science 102 (2006) 1787-1793.

[6] S. Davarpanah, N.M. Mahmoodi, M. Arami, H. Bahrami, F. Mazaheri, Applied Surface Science 255 (2009) 4171-4176.

[7] C.H. Jou, S.M. Lin, L. Yun, M.-C. Hwang, D.G. Yu, W.L. Chou, J.S. Lee, M.C. Yang, Polymers for Advanced Technologies 18 (2007) 235-239.

[8] Y. Kitkulnumchai, A. Ajavakom, M. Sukwattanasinitt, Cellulose 15 (2008) 599608.

[9] S. Sampaio, P. Taddei, P. Monti, J. Buchert, G. Freddi, Journal of Biotechnology 116 (2005) 21-33.

[10] S. Vílchez, A.M. Manich, P. Jovancic, P. Erra, Carbohydrate Polymers 71 (2008) 515-523.

[11] M. Hosny, J.P.N. Rosazza, Journal of Agricultural and Food Chemistry 50 (2002) 5539-5545.

[12] G.W. Plumb, S. De Pascual-Teresa, C. Santos-Buelga, V. Cheynier, G. Williamson, Free Radical Research 29 (1998) 351-358.

[13] Y. Dobashi, T. Hirano, M. Hirano, Y. Ohkatsu, Journal of Photochemistry and Photobiology A: Chemistry 197 (2008) 141-148.

[14] J.S. Lee, E.J. Kim, D. Chung, H.G. Lee, Colloids and Surfaces B: Biointerfaces 74 (2009) 17-22.

[15] H.L. Ma, S. Kermasha, J.M. Gao, R.M. Borges, X.Z. Yu, Journal of Molecular Catalysis B: Enzymatic 57 (2009) 89-95.

[16] A.M. Osman, K.K.Y. Wong, A. Fernyhough, Enzyme and Microbial Technology 40 (2007) 1272-1279.

[17] M. Kurisawa, J.E. Chung, H. Uyama, S. Kobayashi, Macromolecular Bioscience 3 (2003) 758-764.

[18] F. Sousa, G.M. Guebitz, V. Kokol, Process Biochemistry 44 (2009) 749-756.

[19] A.E. Hagerman, K.M. Riedl, G.A. Jones, K.N. Sovik, N.T. Ritchard, P.W. Hartzfeld, T.L. Riechel, Journal of Agricultural and Food Chemistry 46 (1998) 1887-1892.

[20] S. Camarero, O. García, T. Vidal, J. Colom, J.C.d. Río, A. Gutiérrez, M.J. Martínez, A.T. Martínez, Biotechnology in the Pulp and Paper Industry (2002) 213-222.

[21] C. Valls, M.B. Roncero, Bioresource Technology 100 (2009) 2032-2039.

[22] E. Aracri, A. Fillat, J.F. Colom, A. Gutiérrez, J.C. del Río, Á.T. Martínez, T. Vidal, Bioresource Technology 101 (2010) 8211-8216.

[23] M. Schröder, N. Aichernig, G.M. Gübitz, V. Kokol, Biotechnology Journal 2 (2007) 334-341.

[24] K.M.G. Hossain, M.D. González, G.R. Lozano, T. Tzanov, Journal of Biotechnology 141 (2009) 58-63.

[25] X. Ren, G. Buschle-Diller, Colloids and Surfaces A: Physicochemical and Engineering Aspects 299 (2007) 15-21.

[26] E. Nugroho Prasetyo, T. Kudanga, W. Steiner, M. Murkovic, G.S. Nyanhongo, G.M. Guebitz, Food Chemistry 118 (2010) 437-444

[27] E. Nugroho Prasetyo, T. Kudanga, W. Steiner, M. Murkovic, G. Nyanhongo, G. Guebitz, Analytical and Bioanalytical Chemistry 393 (2009) 679-687.

[28] K. Weinges, W. Ebert, D. Huthwelker, H. Mattauch, J. Perner, Liebigs' Annalen der Chemie 726 (1969) 114-124.

[29] S. Guyot, J. Vercauteren, V. Cheynier, Phytochemistry 42 (1996) 1279-1288.

[30] W. Xie, P. Xu, Q. Liu, Bioorganic and Medicinal Chemistry Letters 11 (2001) 1699-1701.

[31] C. Xue, G. Yu, T. Hirata, J. Terao, H. Lin, Bioscience, Biotechnology, and Biochemistry 62 (1998) 206-209.

[32] K.W. Kim, R.L. Thomas, Food Chemistry 101 (2007) 308-313.

[33] P.J. Park, J.Y. Je, S.K. Kim, Carbohydrate Polymers 55 (2004) 17-22.

[34] T. Cushnie, A. Lamb, International Journal of Antimicrobial Agents 113 (2005) 343-356.

[35] D.V. Ratnam, D.D. Ankola, V. Bhardwaj, D.K. Sahana, M.N.V.R. Kumar, Journal of Controlled Release 113 (2006) 189-207.

[36] H. Gradiaiar, P. Pristovaiek, A. Plaper, R. Jerala, Journal of Medicinal Chemistry 50 (2006) 264-271.

[37] S. Tadakatsu, Z. Wei-Hua, H. Zhi-Oing, Anti-Infective Agents in Medicinal Chemistry 6 (2007) 57-62. 